\title{
EFFECT OF SEED PRIMING ON INFECTION WITH WHITE TIP NEMATODE (Aphelenchoides besseyi), SEED-BORNE FUNGI, RICE YIELD, AND YIELD COMPONENTS IN EGYPT. EI-Shafey, R.A.S. ${ }^{1}$ and Rabab M. A. Elamawi ${ }^{2}$ \\ 1- Rice Res. Dept., Field Crops Res. Institute, Agric. Res. Center, Sakha, Kafr El-Sheikh 33717, Egypt. \\ 2- Rice Res. Pathol. Dept., Plant Pathol. Res., Institute, Agric. Res. Center, Sakha, Kafr El-Sheikh 33717, Egypt. \\ Corresponding author Email: relshafey13@ yahoo.com
}

\begin{abstract}
Rice is the second staple food crop after wheat in Egypt. White tip disease of rice leaves induced by the rice leaf nematode, Aphelenchoides besseyi (Christie, 1942), is widespread nearly in all rice ecosystems all over the world, causing remarkable yield reduction to susceptible cultivars. The losses reach to $47 \%$ in Egyptian rice Reiho cultivar. Rice seeds are known as the main source of the disease. The present study was conducted to evaluate the effect of seed priming on white tip nematode infection in rice and seed-borne fungi at different plant growth stages. Severely-infected Reiho rice seeds were primed for $48 \mathrm{hr}$ in solutions of $\mathrm{NaCl}, \mathrm{CaCl}_{2}$, $\mathrm{Na}_{2} \mathrm{CO}_{3}, \mathrm{MgSO}_{4}, \mathrm{CuSO}_{4}$ and micronized sulfur, separately or in mixtures of two salts except for the sulfur treatment. The primed seeds and the corresponding control nonprimed counterparts were then rinsed for three times using tap water and incubated at $30^{\circ} \mathrm{C}$ to asses the germination percentage, which ranged between 96 to $100 \%$ with no significant difference between the primed and non-primed seed treatments. In the lab testing, the maximum seedling vigor index and root / shoot ratio were obtained using seedlings raised from seeds primed with $5 \mathrm{~g} / \mathrm{L}$ solutions of sulfur ( $80 \% \mathrm{WP}$ ) followed by sulfur $+\mathrm{CaCl}_{2}, \mathrm{NaCl}$ and $\mathrm{CuSO}_{4}$. At nursery level, root/shoot ratio increased with all primed seed treatments except with those treated with $\mathrm{NaCl}$ solution. Two field experiments were conducted during the 2007 and 2008 rice growing seasons. All seed priming treatments significantly enhanced most of the agronomic traits and increased yield in comparison with the non-primed infected or healthy seeds. Soaking in sulfur solution at the concentration of $5 \mathrm{~g} / \mathrm{L}$ and its combinations with the other salts at half dose of each gave the highest mortality of white tip nematode, lowest percentage of white tip infection, and least disease severity. Sulfur and $\mathrm{NaCl}$ is then proved to be the most effective priming mixture that can reduce white tip nematode infection. For seedborne fungi, the treatment with $\mathrm{CaCl}_{2}$ alone or in its all combinations has positive effects in elimination of the Fusarium Moniliforme, Helminthosporium oryzae and Alternaria padwickii in comparison with the water treatment control. $\mathrm{CaCl}_{2}$ completely eradiated $F$. moniliforme. Seed treatment with mixtures of salts reduced the fungal growth more than did one salt alone. Generally, all seeds primed with salts showed lower levels of infection with $F$. moniliforme comparing with the control. The treatment with $\mathrm{CuSO}_{4}$ solution at $1.5 \mathrm{~g} / \mathrm{L}$ gave complete protection from seed-borne fungi.

Keywords: Seed priming, $\mathrm{NaCl}, \mathrm{CaCl}_{2}$, Sulfur, white tip nematode, Aphelenchoides besseyi, bakanae, Fusarium Moniliforme, Bipolaris, Alternaria.
\end{abstract}




\section{INTRODUCTION}

Rice is the second important cereal crop in the world. It is essential for $54 \%$ of the world's population. In Egypt, rice is the second staple food after wheat. It is annually grown in more than one million feddan $(420,000 \mathrm{ha})$, mostly in the Northern part of the Nile delta, which produces about 6 million tons of paddy rice/year, with an average of about 10.06 tons/hectare (RRTC, 2009). In the near future, this quantity will not be sufficient because of the dramatic increase in local population and constraints due to many abiotic and biotic factors.

White tip disease of rice leaves caused by the rice leaf nematode, Aphelenchoides besseyi (Christie, 1942), is widespread and found nearly in all different world rice ecosystems, (Ou, 1985). Giudici et al. (2003) and Rajan and Lal (2006) reported that $A$. besseyi is a seed transmitted plant parasitic nematode that can dramatically affect rice growth and yield. Abdel Hadi et al. (2005) investigated $A$. besseyi damage, infection, dynamic symptoms and epidemic features in different locations. They found that the disease is widely spread all over Egypt governorates, causing remarkable yield reduction in the susceptible old rice cultivars Giza 171 and Reiho in addition to the new rice cultivars Giza 177, Sakha 102 and Sakha 103 with different levels of infection and yield losses in old cultivars, reaching $47 \%$ as recorded later by El-Shafey (2007), who studied also the effects of seed priming for $48 \mathrm{hr}$ with $\mathrm{NaCl}$ solutions at the concentrations of 25, 35 and 50 $\mathrm{g} / \mathrm{L}$ and sulfur at $1.5,3$ and $5 \mathrm{~g} / \mathrm{L}$ on rice seeds. The results indicated that soaking in $\mathrm{NaCl}$ solution at $50 \mathrm{~g} / \mathrm{L}$ and sulfur at $5 \mathrm{~g} / \mathrm{L}$ completely killed the white tip nematode juveniles in highly infected seeds without adverse action on seed viability. Moreover, $\mathrm{NaCl}$ and sulfur solutions enhanced seedling vigor and increased plant height and the yield and its components. Quick seedling emergence and even stands are essential for maximizing the yield of all crops (Ashraf and Foolad, 2005 and Yamauchi and Winn, 1996).

Seed infection with stackburn disease caused by Alternaria padwickii (Ganguly) Ellis, causes reduction in germination, seed rotting, rotting of roots and coleoptiles, and death. The fungus usually penetrates into the endosperm and can reduce quality of rice. Brown spot disease caused by Bipolaris oryzae (Breda de Haan) Shoemaker (syn. Helminthosporium oryzae Breda de Haan causes blight of seedlings grown from heavily infected seeds and mortality can reach $58 \%(\mathrm{Ou}, 1985)$. Rice bakanae, the disease is seedborne, also called foolish seedling disease, caused by Fusarium moniliforme Sheldon, is a major disease at seedling stage of rice, spreading all over the world but mostly occurring in Asia. Seedlings infected by the fungus elongate and become weak with yellowish-green leaves and scatter throughout the field. Severely Infected seedlings in the seedbed die before transplanting, and those that survive may die after transplanting, (Ou, 1985; Rood, 2004). In Egypt, it was first recorded in 2000 on the semi-dwarf rice cultivar, Sakha 101, seedbeds in Elhamra and Ezbet Elfar, Kafr El-Sheikh governorate. Then, it was observed in scattered fields of Sakha 101 and Giza 177 cultivars 
(RRTC, 2008). The seed-borne pathogens; B. oryzae, A. padwickii and F. moniliforme caused lower germination of seeds and transmitted diseases from seeds to rice plants (Huynh et al., 2001). The use of high quality, disease-free seeds is essential for good stands and strong seedlings that can emerge rapidly, tolerate adverse weather conditions and resist seed-borne diseases.

Seed priming is a technique in which seeds are partially hydrated until the germination process begins while radical emergence does not occur (Bradford, 1986). Priming activates the metabolic processes necessary for germination to occur but without actual germination (Farooq et al., $2005 \mathrm{a}$ ).

Osmoconditioning or osmopriming is the term used to describe soaking of seeds in aerated, low-water-potential solutions. In this special type of seed priming, polyethylene glycol (PEG) or salt solution is used to control water uptake and prevents radical protrusion (Bray, 1995). The salts used to lower water potential were $\mathrm{KNO}_{3}, \mathrm{KCl}, \mathrm{K}_{3} \mathrm{PO}_{4}, \mathrm{KH}_{2} \mathrm{PO}_{4}, \mathrm{MgSO}_{4}, \mathrm{CaCl}_{2}, \mathrm{NaCl}$, and mannitol. Salts supply the seeds with $\mathrm{N}$ and other nutrients needed for protein synthesis during germination (Farooq et al., 2005 b).

Seed priming has been used to enhance rapid emergence of roots and shoots, more vigorous plants, better drought tolerance in rice (Lee and Kim, 1999, 2000; Basra et al., 2003, 2004, 2005; Farooq et al., 2004, 2005a,b, $2006 \mathrm{a}, \mathrm{b})$ and reduce the incidence of soil- or seed-borne diseases, mainly caused by collar rot (Sclerotium rolfsii) and Fusarium spp., Also, it increases nodulation by native rhizobia (Musa et al., 2001). Several studies have reported that seed priming improved the performance of direct seeded rice, uniform and vigorous germination and rapid emergence (1-3 days), leading to a wide range of phonological and yield-related benefits. On-farm seed priming resulted in better emergence, earlier flowering, taller plants, longer panicles, and more panicles per plant (Harris, et al., 2002; Du and Tuong, 2002; Farooq et al., 2006c,d). However, few studies were conducted to explore the possibility of improving the performance of transplanting rice culture by seed priming (Farooq et al., 2009). Osmopriming with $\mathrm{CaCl}_{2}$ and $\mathrm{CaCl}_{2}+\mathrm{NaCl}$ improved seedling vigor index and seedling and stand establishment in flooded rice (Bray, 1995; Du and Tuong, 2002 ; Ruan et al., 2002). In a field trial, wheat seeds soaked in $1 \%$ sodium carbonate and $5 \%$ sodium bicarbonate solutions for $30 \mathrm{~min}$. and $5 \% \mathrm{NaCl}$ solution for $24 \mathrm{~h}$, increased the number of ear bearing tillers $\mathrm{m}^{-2}$, tillers and enhanced grain yield (Singh \& Gill, 1988; Ashraf et al., 1999).

The beneficial effect of seed priming is primarily due to preenlargement of the embryo (Bradford et al., 1990), biochemical changes like enzyme activation, faster production of emergence metabolites (Lee and Kim, 2000; Basra et al., 2005) and genetic and structural repair (Dell'Aquilla and Tritto, 1990).

The current misuse of nematicides to manage the white tip nematode has hazardous effects on humans and animals and pollutes the environment. Because nematicides have a residual effect, this study aimed to find natural, safe, and available compounds to control white tip nematode disease in rice and seed-borne fungi, in parallel with enhancing the performance of rice in transplanted rice culture and consequently increase grain yield. 


\section{MATERIALS AND METHODS}

Field experiments:

Two field experiments were conducted during the rice seasons 2008 and 2009 at the experimental farm of Rice Research and Training Center (RRTC), Sakha, Kafr El-Sheikh, Egypt. The aim was to investigate the effect of different seed priming treatments of Rieho cultivar (highly susceptible) on management of rice white tip nematode disease, seedling vigor, yield and its components. The treatments were arranged in a randomized complete block design with four replications.

Seed Treatment: A series of experiments were conducted to optimize the different priming strategies for the tested rice variety. Highly infected seeds of Reiho were soaked in water for $48 \mathrm{hr}$ at the rate of $60 \mathrm{~kg}$ seeds $/ 100 \mathrm{~L}$ water/feddan. The seed treatments are described in Table (1). The soaking period was $48 \mathrm{hr}$ for all treatments. After this period, treated seeds were rinsed with tap water for several times to eliminate salt residuals. The soaked seeds were incubated for additional $48 \mathrm{hr}$ under $28^{\circ} \mathrm{C}$ before sowing directly in the nursery.

Table (1): Seed priming treatments.

\begin{tabular}{|c|c|c|c|c|}
\hline No. & Treatment & Dosage g/L & $\mathrm{Ec} \mathrm{ms/m}$ & $\mathrm{pH}$ \\
\hline & \multicolumn{4}{|l|}{ Osmohardening for $48 \mathrm{hr}$} \\
\hline 1 & $\mathrm{NaCl}$ & 50 & 65.0 & 7.59 \\
\hline 2 & $\mathrm{CaCl}_{2}$ & 50 & 63.3 & 7.28 \\
\hline 3 & $\mathrm{NaCO}_{3}$ & 50 & 43.8 & 10.60 \\
\hline 4 & $\mathrm{MgSO}_{4}$ & 50 & 18.4 & 9.50 \\
\hline 5 & $\mathrm{CuSO}_{4}$ & 1.5 & 1.91 & 7.34 \\
\hline 6 & $\mathrm{CuSO}_{4}$ & 2.5 & 3.22 & 6.58 \\
\hline 7 & $\mathrm{NaCl}+\mathrm{CaCl}_{2}$ & $25+25$ & $\overline{64.0}$ & 7.37 \\
\hline 8 & $\mathrm{NaCl}+\mathrm{NaCO}_{3}$ & $25+25$ & 61.2 & 10.43 \\
\hline 9 & $\mathrm{CaCl}_{2}+\mathrm{NaCO}_{3}$ & $25+25$ & 58.0 & 9.66 \\
\hline 10 & $\mathrm{NaCl}+\mathrm{MgSO}_{4}$ & $25+25$ & 58.4 & 8.84 \\
\hline 11 & $\mathrm{CaCl}_{2}+\mathrm{MgSO}_{4}$ & $25+25$ & 54.1 & 6.80 \\
\hline 12 & $\mathrm{MgSO}_{4}+\mathrm{NaCO}_{3}$ & $25+25$ & 21 & 10.25 \\
\hline \multicolumn{5}{|c|}{ Sulfur priming for $48 \mathrm{hr}$} \\
\hline 13 & Sulfur $80 \% W P$ & 5 & 0.4 & 8.30 \\
\hline 14 & Sulfur $80 \% W P$ & 10 & 0.6 & 9.52 \\
\hline 15 & Sulfur $+\mathrm{NaCl}$ & $2.5+25$ & 31.8 & 6.65 \\
\hline 16 & Sulfur + $\mathrm{CaCl}_{2}$ & $2.5+25$ & 30.9 & 6.80 \\
\hline 17 & Sulfur+NaCO & $2.5+25$ & 10.5 & 10.03 \\
\hline 18 & Sulfur+MgSO & $2.5+25$ & 9.1 & 10.65 \\
\hline 19 & Mocap (nematicide) & 2 & -- & -- \\
\hline \multicolumn{5}{|c|}{ Hydropriming for $48 \mathrm{hr}$ (soaking in water; traditional method) } \\
\hline 20 & Healthy grains (control-healthy) & Tap water & 0.7 & 7.32 \\
\hline 21 & Infected grain (control-infected) & Tap water & 0.7 & 7.32 \\
\hline
\end{tabular}

Nursery raising: The treated seeds were sown on 15 May of 2007 and 2008 seasons in the nursery. Fertilizers (N, P and $\mathrm{Zn}$ ) were applied at 15, 9 and $2 \mathrm{~g} \cdot \mathrm{m}^{-2}$, respectively, as urea $(46 \% \mathrm{~N})$, single super phosphate $\left(18 \% \mathrm{P}_{2} \mathrm{O}_{5}\right)$ and $\mathrm{ZnSO}_{4}(35 \% \mathrm{Zn})$. 
Crop husbandry: One month after seed broadcasting, three seedlings/hill were transplanted in plots measured $3 \times 3.5 \mathrm{~m}^{2}$ at $20 \times 20 \mathrm{~cm}$. Fertilizer materials were applied as urea $(46 \% \mathrm{~N})$ at the rate of $144 \mathrm{~kg} \mathrm{~N} / \mathrm{ha}$ in two doses, single super phosphate $\left(18 \% \mathrm{P}_{2} \mathrm{O}_{5}\right)$, and potassium sulfate $(48 \%$ $\mathrm{K}_{2} \mathrm{O}$ ). The whole quantity of phosphorus, potash and zinc, and two thirds of nitrogen were applied and incorporated into the soil as basal fertilizer during seed bed preparation. The remaining third of nitrogen was applied at tillering stage (thirty days after transplanting). Submerged water level at the time of transplanting was $3-4 \mathrm{~cm}$ and $5-6 \mathrm{~cm}$ after one week and till the end of the experimental period.

Seedling characteristics: At the laboratory, 25 infected seeds and 25 primed-infected-seeds were incubated at $28^{\circ} \mathrm{C}$ in $12-\mathrm{cm}$-diameter Petri dishes between two layers of moist filter papers and replicated four times. Seed germination percentage was calculated. Seedling dry weight, root and shoot length were measured 15 days later. Also, the infection (\%) of each seed-borne fungus was calculated in each treatment. All associated fungi were identified. At the nursery, root and shoot length was measured in ten randomly collected seedlings in each replication, 25 days after sowing, then shoot/root ratio was calculated. Seedling vigor index was calculated as germination (\%) $\times$ dry weight (Farooq et al., 2004).

Agronomic traits: Data regarding (plant height, number of tillers and panicles $/ \mathrm{m}^{2}$ ) were taken at the onset of physiological maturity. Data on other yield components, kernel characteristics were taken at full maturity. The yield was calculated as $\mathrm{t} / \mathrm{ha}$. Flag leaf area $\left(\mathrm{cm}^{2}\right)$ was measured using a leaf area meter. The plant height and flag leaf area were used as indicators for white tip nematode infection.

Nematode mortality: The extracted nematodes from seeds were counted and identified with the microscope to calculate mortality as follows:

$$
\text { Nematode mortality }(\%)=\frac{\text { No. of dead nematodes }}{\text { Total no. of nematodes }} \times 100
$$

Disease assessment: Percentage and severity of white tip infection were taken after the full appearance of white tip nematode symptoms. Percentage of white tip nematode infection was calculated according to this formula:

$$
\text { Percentage of infection }=\frac{\text { No. of infected hills } / \mathrm{m}^{2}}{\text { Total no. of rice hills } / \mathrm{m}^{2}} \times 100
$$
leaves $/ \mathrm{m}^{2}$

Severity of white tip infection was estimated as the number of infected

Statistical analysis: Data were subjected to analysis of variance (Gomez and Gomez, 1984), and means significantly differ were compared according to Duncan's Multiple Range Test (Duncan, 1955). 


\section{RESULTS}

Seedling characteristics under laboratory and nursery conditions:

Under laboratory conditions, seed germination and seedling vigor index obtained from primed seeds did not significantly differ from those obtained from non-primed, nematode-infected seeds (Fig.1 and Table 2). The shoot/root ratio were significantly enhanced with the sulfur $5 \mathrm{~g} / \mathrm{L}$ followed by $\mathrm{NaCl}+\mathrm{MgSO}_{4}$ (1.99 and 1.89, respectively) (Table 2).

Under nursery conditions, many of the tested seed priming solutions significantly enhanced shoot length comparing to the non-primed, healthy and infected grains. The highest shoot and root lengthes were recorded for the mixture of sulfur $+\mathrm{NaCl}(22.4$ and $9.5 \mathrm{~cm}$, respectively) and sulfur $(5 \mathrm{~g} / \mathrm{L})$ alone (21.2 and $9.4 \mathrm{~cm}$, respectively) (Table 2). All mixtures of sulfur with salts enhanced the shoot length over the infected seeds. The highest root/shoot ratio was obtained from priming with the combination of $\mathrm{NaCl}+\mathrm{MgSO}_{4}$ (0.51), whereas, the lowest ratio was obtained from the treatment with $\mathrm{NaCl}$ without significant differences with infected and healthy non-primed seeds $(0.33,0.36$ and 0.39$)$ (Table 2$)$.

Table (2): Influence of seed priming on rice seedling vigor under laboratory and nursery conditions.

\begin{tabular}{|c|c|c|c|c|c|c|c|}
\hline \multirow[b]{2}{*}{ No. } & \multirow[b]{2}{*}{ Treatment } & \multicolumn{3}{|c|}{ Laboratory } & \multicolumn{3}{|c|}{ Nursery conditions (15 DAS)* } \\
\hline & & $\begin{array}{c}\text { Germination } \\
(\%)\end{array}$ & $\begin{array}{l}\text { Seedling } \\
\text { vigor index }\end{array}$ & $\begin{array}{c}\text { Root/ } \\
\text { shoot } \\
\text { ratio }\end{array}$ & $\begin{array}{l}\text { Shoot } \\
\text { length } \\
\text { (cm) }\end{array}$ & $\begin{array}{c}\text { Root } \\
\text { length } \\
\text { (cm) }\end{array}$ & $\begin{array}{l}\text { Root/ } \\
\text { shoot } \\
\text { ratio }\end{array}$ \\
\hline & \multicolumn{7}{|l|}{ Osmohardening } \\
\hline 1 & $\mathrm{NaCl}$ & 98.87 & 11.41 & $1.74 \mathrm{abcd}$ & $17.7 \mathrm{~d}-\mathrm{g}$ & $5.7 \mathrm{k}$ & $0.33 \mathrm{~g}$ \\
\hline 2 & $\mathrm{CaCl}_{2}$ & 97.3 & 10.01 & $1.84 \mathrm{abc}$ & $18.3 \mathrm{c}-\mathrm{f}$ & $7.4 \mathrm{e}-\mathrm{h}$ & 0.41 def \\
\hline 3 & $\mathrm{NaCO}_{3}$ & 97.3 & 10.32 & $1.56 \mathrm{cde}$ & $19.0 \mathrm{~b}-\mathrm{e}$ & $7.2 \mathrm{fgh}$ & 0.38 efg \\
\hline 4 & $\mathrm{MgSO}_{4}$ & 98.7 & 10.84 & $\begin{array}{c}1.72 \\
\text { abcde }\end{array}$ & 18.0 def & $8.0 c-f$ & 0.45 a-e \\
\hline 5 & $\mathrm{CuSO}_{4}(1.5 \mathrm{~g} / \mathrm{L})$ & 100 & 11.41 & $0.62 \mathrm{f}$ & $19.0 \mathrm{~b}-\mathrm{e}$ & $6.8 \mathrm{hij}$ & $0.36 \mathrm{fg}$ \\
\hline 6 & $\mathrm{CuSO}_{4}(2.5 \mathrm{~g} / \mathrm{L})$ & 97.3 & 11.30 & $0.57 \mathrm{f}$ & $17.4 \mathrm{~d}-\mathrm{g}$ & $6.3 \mathrm{ijk}$ & $0.36 \mathrm{fg}$ \\
\hline 7 & $\mathrm{NaCl}+\mathrm{CaCl}_{2}$ & 100 & 10.33 & 1.66 bcde & 18.0 def & $7.0 \mathrm{ghi}$ & 0.39 efg \\
\hline 8 & $\mathrm{NaCl}+\mathrm{NaCO}_{3}$ & 96.0 & 10.67 & 1.69 bcde & $17.6 \mathrm{~d}-\mathrm{g}$ & $8.0 c-f$ & $0.45 a-e$ \\
\hline 9 & $\mathrm{NaCl}+\mathrm{MgSO}_{4}$ & 97.3 & 10.54 & $1.89 \mathrm{ab}$ & $16.3 \mathrm{fg}$ & $8.4 \mathrm{bcd}$ & $0.51 \mathrm{a}$ \\
\hline 10 & $\mathrm{CaCl}_{2}+\mathrm{NaCO}_{3}$ & 98.7 & 10.07 & 1.69 bcde & $16.5 \mathrm{efg}$ & $8.3 \mathrm{~b}-\mathrm{e}$ & $0.50 \mathrm{ab}$ \\
\hline 11 & $\mathrm{CaCl}_{2}+\mathrm{MgSO}_{4}$ & 97.3 & 10.86 & $1.59 \mathrm{cde}$ & $17.3 \mathrm{~d}-\mathrm{g}$ & $7.5 \mathrm{~d}-\mathrm{h}$ & $0.43 b-f$ \\
\hline 12 & $\mathrm{MgSO}_{4}+\mathrm{NaCO}_{3}$ & 97.3 & 9.66 & $1.43 \mathrm{e}$ & $18.5 \mathrm{c}-\mathrm{f}$ & $9.3 \mathrm{a}$ & $0.50 a b$ \\
\hline \multicolumn{8}{|c|}{ Sulfur priming } \\
\hline 13 & Sulfur $5 g$ & 98.7 & 11.54 & $1.99 \mathrm{a}$ & $21.2 \mathrm{ab}$ & $9.4 \mathrm{a}$ & $0.44 \mathrm{a}-\mathrm{e}$ \\
\hline 14 & Sulfur $10 \mathrm{~g}$ & 96.0 & 9.63 & 1.68 bcde & $17.5 \mathrm{~d}-\mathrm{g}$ & $7.6 \mathrm{~d}-\mathrm{h}$ & $0.44 \mathrm{~b}-\mathrm{e}$ \\
\hline 15 & Sulfur $+\mathrm{NaCl}$ & 98.7 & 9.16 & $1.76 \mathrm{abc}$ & $22.4 \mathrm{a}$ & $9.5 \mathrm{a}$ & $0.43 c-f$ \\
\hline 16 & Sulfur $+\mathrm{CaCl}_{2}$ & 98.7 & 11.42 & $1.80 \mathrm{abc}$ & $17.6 \mathrm{~d}-\mathrm{g}$ & $8.7 \mathrm{abc}$ & $0.49 a b c$ \\
\hline 17 & Sulfur $+\mathrm{MgSO}_{4}$ & 98.7 & 9.77 & $1.49 \mathrm{de}$ & $19.9 \mathrm{bcd}$ & $7.7 \mathrm{~d}-\mathrm{g}$ & $0.39 \mathrm{efg}$ \\
\hline 18 & Sulfur $+\mathrm{NaCO}_{3}$ & 97.3 & 10.97 & $1.74 \mathrm{abcd}$ & $20.6 \mathrm{abc}$ & $8.7 \mathrm{abc}$ & $0.43 \mathrm{c}-\mathrm{f}$ \\
\hline \multirow[t]{2}{*}{19} & Mocap & 98.7 & 10.79 & 1.61 bcde & $18.7 \mathrm{c}-\mathrm{f}$ & $9.0 \mathrm{ab}$ & $0.48 \mathrm{a}-\mathrm{d}$ \\
\hline & \multicolumn{7}{|c|}{ Hydropriming for $\mathbf{4 8} \mathrm{hr}$ (soaking in water; traditional method) } \\
\hline 20 & Infected grains & 97.3 & 10.27 & 1.55 cde & $16.3 \mathrm{~g}$ & $6.1 \mathrm{jk}$ & $0.36 \mathrm{fg}$ \\
\hline 21 & Healthy grains & 98.7 & 10.63 & $1.62 \mathrm{bcd}$ & 18.0 def & $7.2 \mathrm{fgh}$ & $0.39 \mathrm{efg}$ \\
\hline & $\mathbf{F}$ & NS & NS & ** & ** & ** & ** \\
\hline
\end{tabular}

In the same column, means followed by same letter(s) are not significantly different at the $5 \%$ level by DMRT. *DAS = days after seeding. 


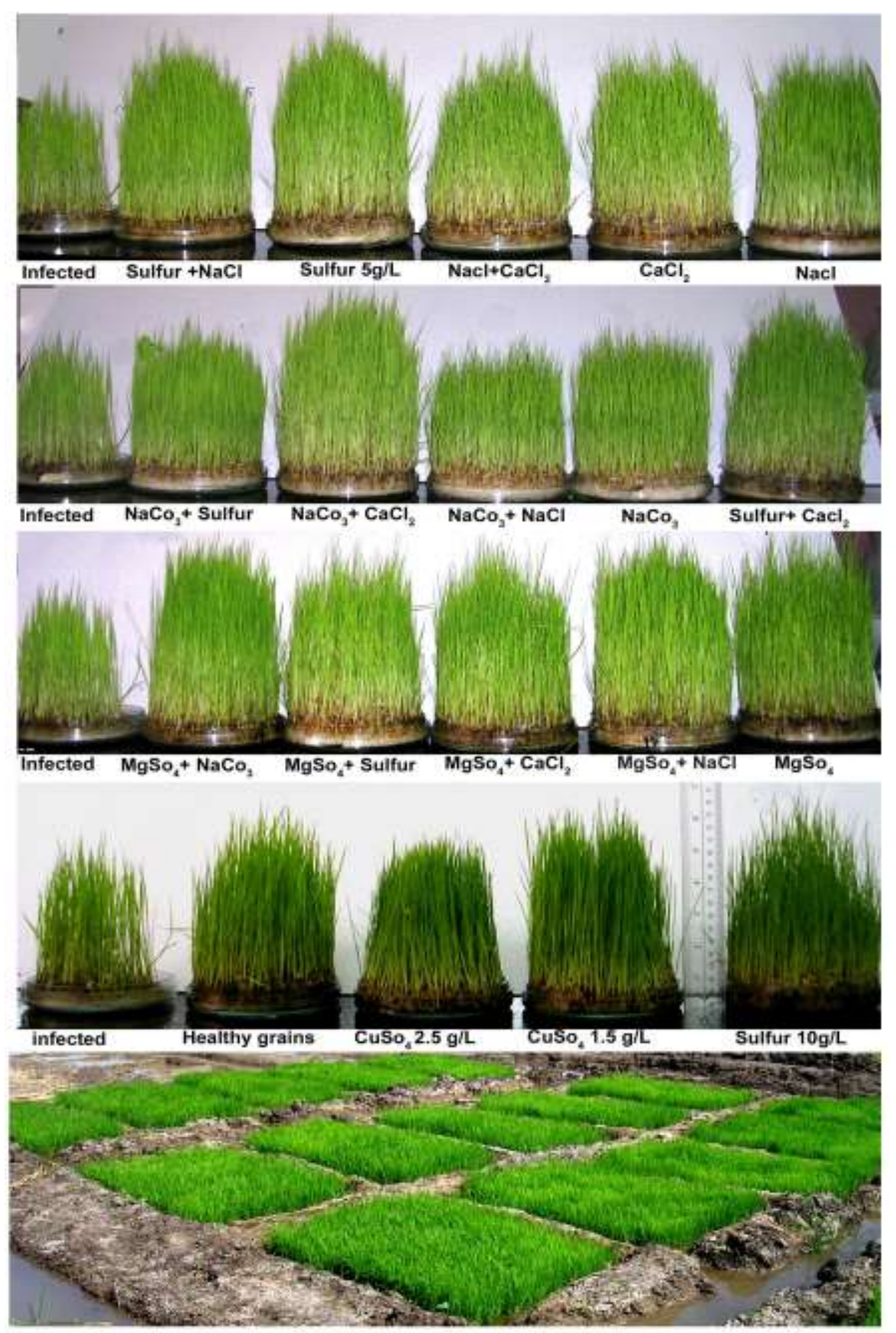

Fig. (1): Effect of seed priming treatments on germination and rice seedlings growth under laboratory conditions (Plates) and at nursery beds. 
Effect of seed priming on agronomic traits of Reiho rice cultivar:

Under field traits, the plant height significantly enhanced by seed priming treatments and decreased by infection with white tip nematode. The highest plants height were obtained with sulfur $5 \mathrm{~g} / \mathrm{L}, \mathrm{NaCl}$, sulfur $+\mathrm{MgSO}_{4}$, and $\mathrm{CaCl}_{2}+\mathrm{MgSO}_{4}(135.2,135.1,135.1,135.0 \mathrm{~cm}$, respectively) (table 3), without no significant differences among them, whereas the shortest plants were obtained from non-primed infected seeds $(112 \mathrm{~cm})$.

The maximum flag leaf area was obtained by seeds primed with the sulfur $5 \mathrm{~g} / \mathrm{L}\left(32.3 \mathrm{~cm}^{2}\right)$ followed by $\mathrm{CaCl}_{2}, \mathrm{NaCl}+\mathrm{CaCl}_{2}$ and then $\mathrm{NaCl}(26.3$, 26.0 and $25.9 \mathrm{~cm}^{2}$, respectively), while the lowest value was recorded for the non-primed infected-seeds $\left(15.1 \mathrm{~cm}^{2}\right)$ Table (3). The value of flag leaf area obtained from nematicide Mocap was similar to the non-primed healthy seeds with no significant differences ( 25.7 and $25.4 \mathrm{~cm}^{2}$ ) (Table 3 and Fig 2).

Number of tillers and panicles bearing tillers were recorded using plants raised from sulfur $5 \mathrm{~g} / \mathrm{L}$ and $\mathrm{NaCl},\left(878.3\right.$ and 818.3 tillers $\left./ \mathrm{m}^{2)}\right)$ and (825.0 and 775.0 panicles $/ \mathrm{m}^{2}$ ), while the lowest number of tillers and panicles were recorded from the non-primed infected seeds 633.3 and 593.3 tillers $/ \mathrm{m}^{2}$ (Table 3).

Concerning, the panicle length and panicle weight, the highest values were recorded with sulfur $5 \mathrm{~g} / \mathrm{L}(23.3 \mathrm{~cm}$ and $4.2 \mathrm{~g})$. Whereas, the lowest values were obtained from the non-primed $(12.9 \mathrm{~cm}$ and $2.2 \mathrm{~g})$ Table (3)

Table (3): Agronomic traits and yield components of transplanted rice grown from different primed seeds.

\begin{tabular}{|c|c|c|c|c|c|c|c|c|}
\hline Treatment & $\begin{array}{c}\text { Plant } \\
\text { height } \\
\text { (cm) }\end{array}$ & $\begin{array}{c}\text { Flag leaf } \\
\text { area } \\
\left(\mathrm{cm}^{2}\right)\end{array}$ & $\begin{array}{l}\text { No. of } \\
\text { tillers } / \mathrm{m}^{2}\end{array}$ & $\begin{array}{c}\text { No. of } \\
\text { panicles/ } \\
\mathrm{m}^{2}\end{array}$ & $\begin{array}{c}\text { Panicle } \\
\text { length } \\
\text { (cm) }\end{array}$ & $\begin{array}{c}\text { Panicle } \\
\text { weight } \\
\text { (g) }\end{array}$ & $\begin{array}{l}1000- \\
\text { Grain } \\
\text { weight } \\
\text { (g) }\end{array}$ & $\begin{array}{l}\text { Yield } \\
\text { (t/ha) }\end{array}$ \\
\hline \multicolumn{9}{|l|}{ Osmohardening } \\
\hline $\mathrm{NaCl}$ & $135.1 \mathrm{a}$ & $25.9 b$ & 818.3ab & 775.0ab & 22.9ab & 3.9ab & $26.2 a$ & $8.910 \mathrm{a}$ \\
\hline $\mathrm{CaCl}_{2}$ & $128.7 \mathrm{~cd}$ & $26.3 b$ & 765.7abc & 733.3a-c & $22.0 \mathrm{bcd}$ & $3.5 c-f$ & $25.0 \mathrm{bcd}$ & $8.267 \mathrm{bcd}$ \\
\hline $\mathrm{NaCO}_{3}$ & $121.5 \mathrm{fg}$ & $23.0 \mathrm{e}$ & 633.3bcd & $585.0 c-f$ & $20.8 \mathrm{fg}$ & 3.5c-f & 24.8abc & $7.619 \mathrm{~d}-\mathrm{i}$ \\
\hline $\mathrm{MgSO}_{4}$ & $130.5 \mathrm{a}-\mathrm{d}$ & 23.7cde & 671.7bcd & 625.0c-f & 20.3gh & $3.5 c-f$ & 24.5bcd & $7.429 \mathrm{e}-\mathrm{i}$ \\
\hline $\mathrm{CuSO}_{4}(1.5 \mathrm{~g} / \mathrm{L})$ & $128.7 \mathrm{~cd}$ & $18.7 f$ & 538.3de & $516.7 \mathrm{e}-\mathrm{g}$ & $19.2 \mathrm{i}$ & 2.9gh & $22.4 \mathrm{e}$ & $6.705 \mathrm{hij}$ \\
\hline $\mathrm{CuSO}_{4}(2.5 \mathrm{~g} / \mathrm{L})$ & 122.5ef & $20.0 f$ & 517.0de & $429.5 f g$ & $19.0 \mathrm{i}$ & $2.6 \mathrm{~h}$ & 23.2de & $6.514 \mathrm{ij}$ \\
\hline $\mathrm{NaCl}+\mathrm{CaCl}_{2}$ & $128.4 \mathrm{~cd}$ & 26.0b & 768.3abc & 685.0a-d & 21.9bcd & $3.8 \mathrm{bc}$ & 24.0cde & $7.467 e-i$ \\
\hline $\mathrm{NaCl}+\mathrm{NaCO}_{3}$ & $120.2 \mathrm{fg}$ & 23.9cde & 733.3abc & 621.7c-f & 21.4def & 3.5c-f & $25.4 a b$ & 8.076b-f \\
\hline $\mathrm{NaCl}+\mathrm{MgSO}_{4}$ & 132.6abc & $19.9 f$ & 610.0cde & 595.0c-f & 20.2gh & $3.2 \mathrm{fg}$ & $22.4 \mathrm{e}$ & $6.857 \mathrm{ghi}$ \\
\hline $\mathrm{CaCl}_{2}+\mathrm{NaCO}_{3}$ & $117.7 \mathrm{~g}$ & $19.5 f$ & 640.0bcd & 570.0def & 19.3hi & $3.0 \mathrm{~g}$ & $22.6 \mathrm{e}$ & $7.124 f-i$ \\
\hline $\mathrm{CaCl}_{2}+\mathrm{MgSO}_{4}$ & $135.0 \mathrm{a}$ & $22.9 \mathrm{e}$ & 623.3cde & $605.8 \mathrm{c}-\mathrm{f}$ & 21.4def & $3.4 \mathrm{ef}$ & 23.7cde & $7.238 \mathrm{e}-\mathrm{i}$ \\
\hline $\mathrm{MgSo}_{4}+\mathrm{NaCO}_{3}$ & 131.1a-d & 23.5de & 596.7cde & 558.3def & 20.2gh & $3.4 \mathrm{ef}$ & 25.0abc & $7.086 f-i$ \\
\hline \multicolumn{9}{|c|}{ Sulfur priming } \\
\hline Sulfur $5 g$ & $135.2 \mathrm{a}$ & $32.3 a$ & $878.3 a$ & $825.0 a$ & 23.3a & $4.2 \mathrm{a}$ & $25.8 a$ & $9.371 \mathrm{a}$ \\
\hline Sulfur $10 \mathrm{~g}$ & 126.8de & $22.1 \mathrm{e}$ & $600.0 \mathrm{cde}$ & 565.0def & $22.7 a b$ & 3.7b-e & 23.7cde & $7.314 \mathrm{e}-\mathrm{i}$ \\
\hline Sulfur + NaCl & $130.2 a-d$ & $23.3 e$ & 738.3abc & 685.0a-d & 21.7c-f & 3.6b-e & 24.4bcd & 8.762abc \\
\hline Sulfur $+\mathrm{CaCl}_{2}$ & $129.0 \mathrm{~cd}$ & $25.7 b c$ & 776.7abc & 733.3abc & 21.8b-e & $3.8 \mathrm{bc}$ & 25.0abc & $8.914 a$ \\
\hline Sulfur+MgSO $_{4}$ & $135.1 \mathrm{a}$ & 23.8cde & 591.7cde & 551.7def & 22.7abc & 3.9ab & 24.6bcd & $7.010 f-i$ \\
\hline Sulfur+ $\mathrm{NaCO}_{3}$ & 126.9de & 23.9cde & 651.7bcd & 624.2c-f & 21.6def & 3.6b-e & 24.4bcd & $7.924 b-g$ \\
\hline Mocap & $128.7 \mathrm{~cd}$ & $25.4 \mathrm{bc}$ & 740.0abc & $680.0 \mathrm{a}-\mathrm{d}$ & 22.8ab & 3.8bcd & 24.8abc & 7.733c-h \\
\hline \multicolumn{9}{|c|}{ Hydropriming for $48 \mathrm{hr}$ (soaking in water; traditional method) } \\
\hline Healthy grains & $128.2 \mathrm{~cd}$ & $25.7 \mathrm{bc}$ & $633.3 \mathrm{bcd}$ & $593.3 c-f$ & 20.8efg & 3.5c-f & 25.0 abc & 8.267 bcd \\
\hline Infectedgrains & 112.0h & $15.1 \mathrm{~g}$ & $445.0 \mathrm{e}$ & $405.0 \mathrm{~g}$ & 12.9j & $2.2 i$ & $20.5 \mathrm{f}$ & $5.829 \mathrm{j}$ \\
\hline
\end{tabular}




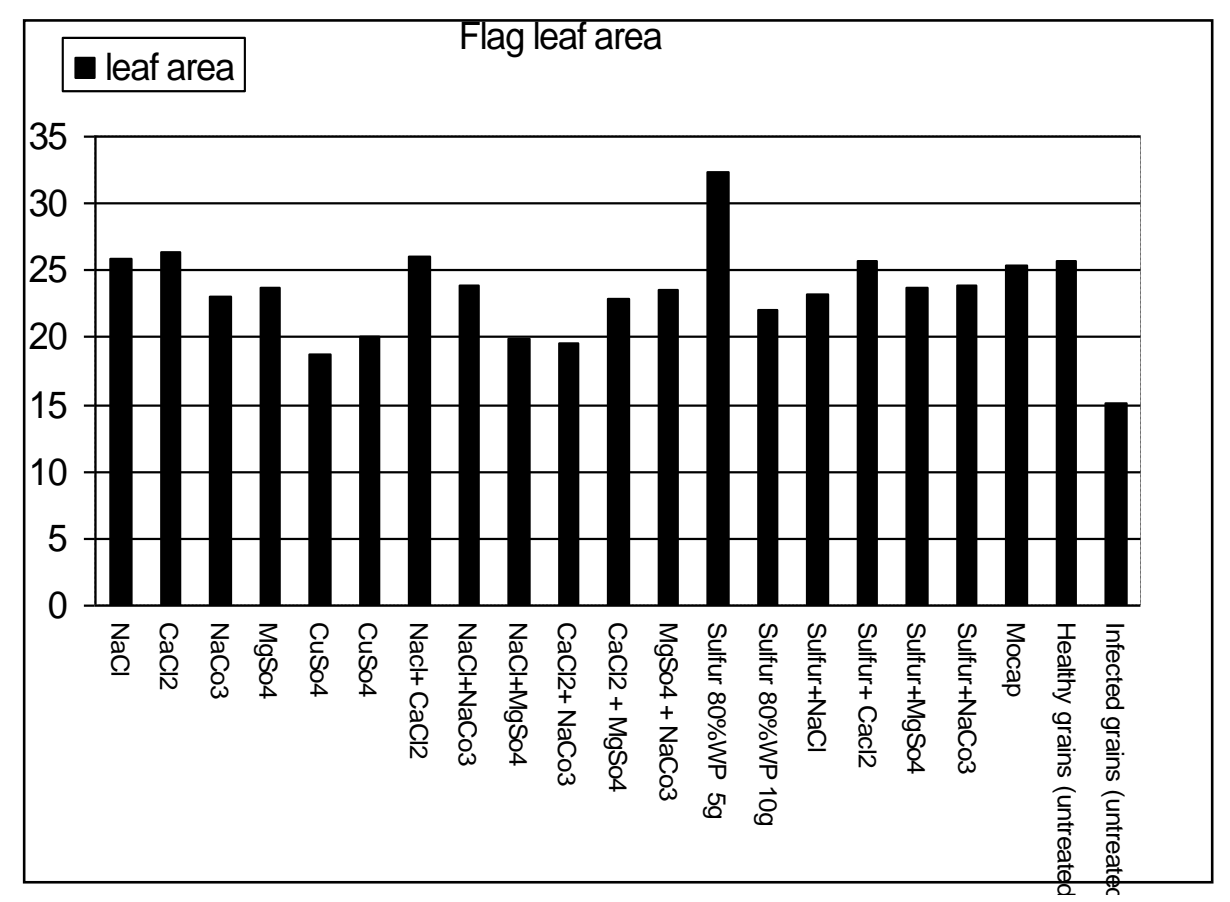

Fig. (2): Effect of different prime-seed treatments on the flag leaf area of transplanted rice.

\section{Effect of seed priming on the yield and yield components:}

In general, all combinations of salts significantly increased grain yield in comparing with the non-primed, infected seeds. Whereas, the highest grain yield was obtained with sulfur $5 \mathrm{~g} / \mathrm{L}$ and $\mathrm{NaCl}$, (9.371 and 8.910, t/ha, respectively). The lowest yield was recorded from the non-primed, infected seeds $(5.829 \mathrm{t} / \mathrm{ha})$. The highest grain filling values were recorded from seeds primed with sulfur $(5 \mathrm{~g} / \mathrm{L})$ and $\mathrm{NaCl}(25.8$ and $26.2 \mathrm{~g}$, respectively), while the lowest value was obtained from the non-primed, infected seeds $(20.5 \mathrm{~g})$ Table (4)

\section{Effect of seed priming on infection with white tip nematode:}

All salts and sulfur significantly increased mortality percentage of white tip nematode (Fig.3 and Table 4). The highest mortality percentage was recorded with soaking in $\mathrm{NaCO}_{3}+\mathrm{NaCl}$, sulfur $5 \mathrm{~g}, \mathrm{NaCO}_{3}$, and Sulfur+ $\mathrm{MgSO}_{4}(97.7,97.3,97.3$, and 97.0 , respectively) with no significant differences among them and between the nematicide treatment Mocap (97.7). The lowest mortality percentage was obtained with soaking in $\mathrm{CuSO}_{4}$ $1.5 \mathrm{~g} / \mathrm{L}$ (44.3) comparing to the infected grain socking in water (traditional methods) (1.0) (Fig. 3 and Table 4). 
El-Shafey, R.A.S. and Rabab M. A. Elamawi

Table (4): Effect of seed priming on white tip nematode infection.

\begin{tabular}{|c|c|c|c|c|}
\hline Treatment & $\begin{array}{c}\text { Mortality } \\
(\%)\end{array}$ & $\begin{array}{c}\text { Infection } \\
(\%)\end{array}$ & $\begin{array}{c}\text { Severity of } \\
\text { infection }\end{array}$ & $\begin{array}{c}\text { Efficiency } \\
(\%)\end{array}$ \\
\hline \multicolumn{5}{|l|}{ Osmohardening } \\
\hline $\mathrm{NaCl}$ & $94.7 \mathrm{bcd}$ & $2.96 \mathrm{gh}$ & $2.33 \mathrm{~d}$ & 96.61 \\
\hline $\mathrm{CaCl}_{2}$ & $94.7 \mathrm{bcd}$ & $4.44 \mathrm{fgh}$ & $3.33 \mathrm{~d}$ & 94.92 \\
\hline $\mathrm{NaCO}_{3}$ & $97.3 \mathrm{a}$ & $1.48 \mathrm{~h}$ & $1.33 \mathrm{~d}$ & 98.31 \\
\hline $\mathrm{MgSO}_{4}$ & $94.3 \mathrm{~cd}$ & $4.44 \mathrm{fgh}$ & $4.00 \mathrm{~d}$ & 94.92 \\
\hline $\mathrm{CuSO}_{4}(1.5 \mathrm{~g} / \mathrm{L})$ & $44.3 \mathrm{j}$ & $34.8 \mathrm{~b}$ & $84.00 \mathrm{~b}$ & 60.19 \\
\hline $\mathrm{CuSO}_{4}(2.5 \mathrm{~g} / \mathrm{L})$ & $56.3 \mathrm{i}$ & $26.6 \mathrm{c}$ & $37.66 \mathrm{c}$ & 69.57 \\
\hline $\mathrm{NaCl}+\mathrm{CaCl}_{2}$ & $94 \mathrm{de}$ & $2.96 \mathrm{gh}$ & $2.00 \mathrm{~d}$ & 96.61 \\
\hline $\mathrm{NaCl}+\mathrm{NaCO}_{3}$ & $97.7 \mathrm{a}$ & $0.74 \mathrm{~h}$ & $0.66 \mathrm{~d}$ & 99.15 \\
\hline $\mathrm{NaCl}+\mathrm{MgSO}_{4}$ & $59.7 \mathrm{~h}$ & $12.59 \mathrm{~d}$ & $24.00 \mathrm{~cd}$ & 85.60 \\
\hline $\mathrm{CaCl}_{2}+\mathrm{NaCO}_{3}$ & $63.6 \mathrm{~g}$ & $11.11 \mathrm{de}$ & $16.33 \mathrm{~cd}$ & 87.29 \\
\hline $\mathrm{CaCl}_{2}+\mathrm{MgSO}_{4}$ & $75.7 \mathrm{f}$ & 8.15 ef & $7.66 \mathrm{~d}$ & 90.68 \\
\hline $\mathrm{MgSO}_{4}+\mathrm{NaCO}_{3}$ & $94.3 \mathrm{~cd}$ & $4.44 \mathrm{fgh}$ & $4.00 \mathrm{~d}$ & 94.92 \\
\hline \multicolumn{5}{|l|}{ Sulfur priming } \\
\hline Sulfur $80 \% W P 5 g$ & $97.3 \mathrm{a}$ & $4.44 \mathrm{fgh}$ & $1.66 \mathrm{~d}$ & 94.92 \\
\hline Sulfur $80 \% W P 10 \mathrm{~g}$ & $93 \mathrm{e}$ & $5.18 \mathrm{fgh}$ & $4.33 \mathrm{~d}$ & 94.07 \\
\hline Sulfur $+\mathrm{MgSO}_{4}$ & $97 \mathrm{a}$ & $0.74 \mathrm{~h}$ & $1.00 \mathrm{~d}$ & 99.15 \\
\hline Sulfur + NaCl & $95.7 \mathrm{~b}$ & $6.66 \mathrm{fg}$ & $6.33 \mathrm{~d}$ & 92.38 \\
\hline Sulfur $+\mathrm{CaCl}_{2}$ & $94.3 \mathrm{~cd}$ & $2.96 \mathrm{gh}$ & $3.00 \mathrm{~d}$ & 96.61 \\
\hline Sulfur $+\mathrm{NaCO}_{3}$ & $95.3 \mathrm{bc}$ & $2.96 \mathrm{gh}$ & $2.00 \mathrm{~d}$ & 96.61 \\
\hline Mocap & $97.7 \mathrm{a}$ & $3.70 \mathrm{fgh}$ & $5.66 \mathrm{~d}$ & 95.77 \\
\hline \multicolumn{5}{|c|}{ Hydropriming for $48 \mathrm{~h}$ (soaking in water; traditional method) } \\
\hline Healthy grains & $1.3 \mathrm{k}$ & $0.70 \mathrm{~h}$ & $0.66 \mathrm{~d}$ & 99.15 \\
\hline Infected grains & $1.0 \mathrm{k}$ & $87.41 \mathrm{a}$ & $234.66 \mathrm{a}$ & \\
\hline
\end{tabular}

In the same column, means followed by the same letter(s) are not significantly different at the $5 \%$ level by DMRT.

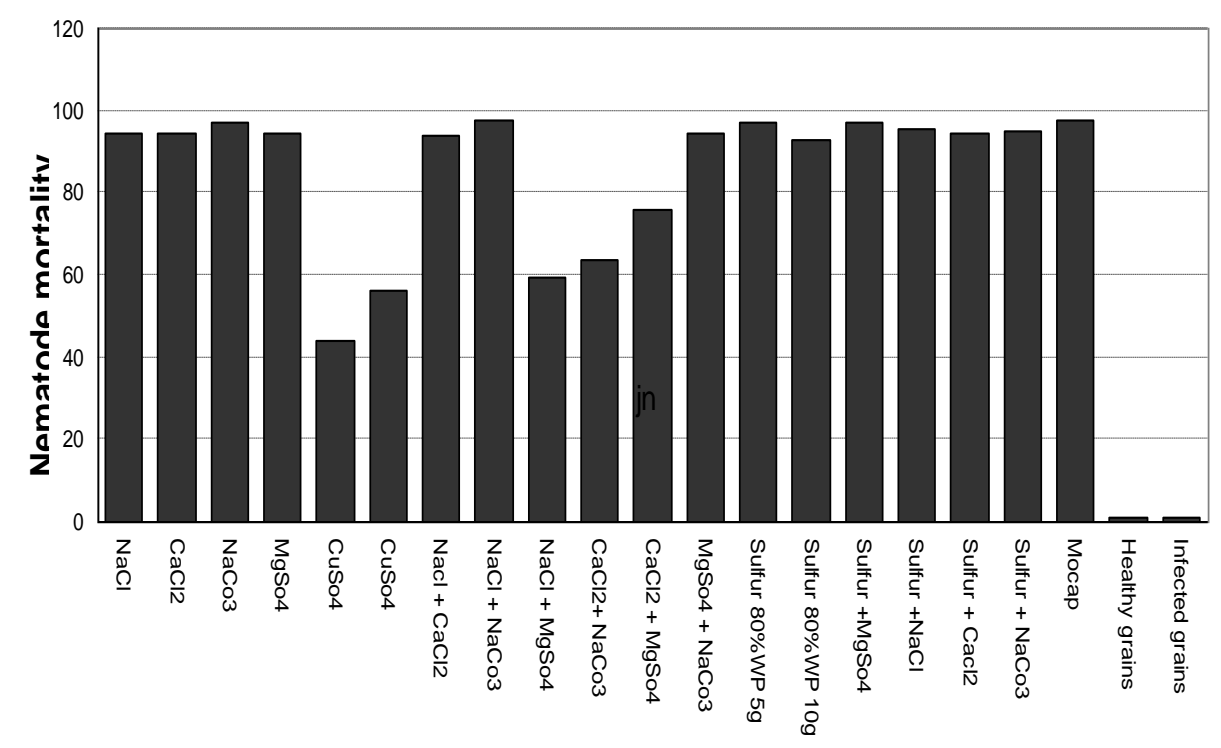

Fig. (3): Effect of seed priming on white tip nematode infection under laboratory conditions. 
In accordance with the nematode mortality results, all seed priming treatments resulted in various levels of nematode infection. The non-primed, infected seeds produced $87.41 \%$ infection. The lowest nematode infection appeared with priming with $\mathrm{NaCO}_{3}, \mathrm{NaCO}_{3}+\mathrm{NaCl}$, and Sulfur $+\mathrm{MgSO}_{4}$ $(1.48,0.74$, and $0.74, \%$, respectively) with no significant differences between them and the non-primed healthy seeds which recorded $0.70 \%$ infection. There is close relation between the mortality (\%), infection (\%), severity of infection (number of infected leaves $/ \mathrm{m}^{2}$ ), and the efficiency of treatment (Table 4 and Fig 4)

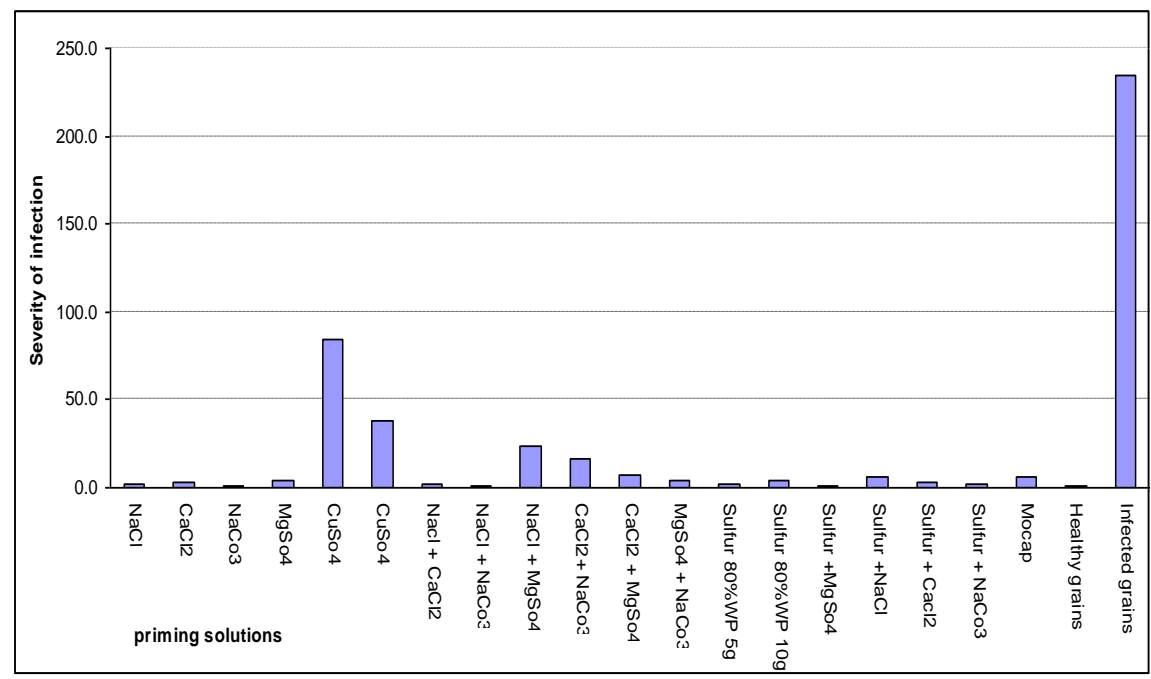

Fig. (4): Effect of seed priming on white tip nematode severity of infection under field conditions.

\section{Effect of seed priming on associated fungi with infected rice seeds by white tip nematode under lab conditions. \\ Under laboratory conditions, twenty five seeds from nematode-infected} and primed-nematode-infected seeds were incubated in Petri dishes at $28^{\circ} \mathrm{C}$. Six days after incubation, seeds were observed using the light microscopy to determine the associated fungi. As shown in (Table 5), Alternaria padwickii, Bipolaris oryzae and Fusarium moniliforme (causal of rice foot rot disease, Bakanae) were found in high percentages, whereas treatment with sulfur and $\mathrm{CuSO}_{4}$ being the best in elimination of the three fungal pathogens on the treated seeds. In contrast, treatment with $\mathrm{NaCl}$ and $\mathrm{CaCl}_{2}$ increased the incidence of $A$. padwickii and $B$. oryzae, while the treatment with $\mathrm{CaCl}_{2}$ alone or in combinations with other salts (except with $\mathrm{NaCO}_{3}$ ) completely eliminated $F$. moniliforme (Table 5). Seeds treated with mixtures of different salts reduced the occurrence of fungi more than did one salt alone. In general, all seeds primed with salts showed reduction in the incidence of $F$. moniliforme comparing with the control (Table 5). 
Table (5): Effect of seed priming on incidence of pathogenic fungi on rice seeds under laboratory conditions.

\begin{tabular}{|c|c|c|c|c|}
\hline \multirow{3}{*}{ No. } & \multirow[b]{2}{*}{ Treatment } & \multicolumn{3}{|c|}{ Incidence (\%) } \\
\hline & & $\begin{array}{l}\text { Alternaria } \\
\text { padwickii }\end{array}$ & Bipolaris oryzae & $\begin{array}{c}\text { Fusarium } \\
\text { moniliforme }\end{array}$ \\
\hline & Osmohardening & & & \\
\hline 1 & $\mathrm{NaCl}$ & 72 & 84 & 15 \\
\hline 2 & $\mathrm{CaCl}_{2}$ & 76 & 78 & 0 \\
\hline 3 & $\mathrm{NaCO}_{3}$ & 32 & 32 & 24 \\
\hline 4 & $\mathrm{MgSO}_{4}$ & 40 & 16 & 20 \\
\hline 5 & $\mathrm{CuSO}_{4}(1.5 \mathrm{~g} / \mathrm{L})$ & 8 & 1 & 0 \\
\hline 6 & $\mathrm{CuSO}_{4}(2.5 \mathrm{~g} / \mathrm{L})$ & 0 & 0 & 0 \\
\hline 7 & $\mathrm{NaCl}+\mathrm{CaCl}_{2}$ & 50 & 52 & 0 \\
\hline 8 & $\mathrm{NaCO}_{3}+\mathrm{NaCl}$ & 0 & 36 & 31 \\
\hline 9 & $\mathrm{NaCO}_{3}+\mathrm{CaCl}_{2}$ & 0 & 64 & 16 \\
\hline 10 & $\mathrm{MgSO}_{4}+\mathrm{NaCl}$ & 0 & 40 & 12 \\
\hline 11 & $\mathrm{MgSO}_{4}+\mathrm{CaCl}_{2}$ & 8 & 24 & 0 \\
\hline \multirow[t]{2}{*}{12} & $\mathrm{MgSO}_{4}+\mathrm{NaCO}_{3}$ & 0 & 44 & 24 \\
\hline & Sulfur priming & & & \\
\hline 13 & Sulfur $80 \% W P \quad 5 g / l$ & 68 & 0 & 13 \\
\hline 14 & Sulfur $80 \% W P \quad 10 \mathrm{~g} / \mathrm{l}$ & 0 & 56 & 16 \\
\hline 15 & Sulfur + NaCl & 28 & 52 & 8 \\
\hline 16 & Sulfur $+\mathrm{CaCl}_{2}$ & 60 & 68 & 0 \\
\hline 17 & $\mathrm{NaCO}_{3}+$ Sulfur & 0 & 40 & 8 \\
\hline \multirow[t]{2}{*}{18} & $\mathrm{MgSO}_{4}+$ Sulfur & 0 & 56 & 24 \\
\hline & \multicolumn{4}{|c|}{ Hydropriming (soaking in water; traditional method) } \\
\hline 19 & Control & 49 & 80 & 52 \\
\hline & L.S.D. 5\% & 1.154 & 0.611 & 0.791 \\
\hline
\end{tabular}

\section{DISCUSSION}

Seed priming under laboratory conditions had no effect on rice seed germination and rather slightly improved seedling vigor index, shoot/root ratio comparing with the traditional method of seed soaking in water (hydropriming). The improvement effect of seed priming technique was recognizable after 20 days from sowing comparing with the traditional method. $\mathrm{MgSO}_{4}$ was the most effective treatment, alone or in combination with other salts, followed by the priming with sulfur.

It was revealed from the present study that priming technique can enhance seedling establishment and rice yield and can also reduce nematode infection in the rice field with a positive turnover effect on crop productivity.

In general, osmohardining and hydropriming techniques enhance seed germination likely by their role in enzyme activation, in particular those involved in hydrolases. This is plausible as a positive correlation exists between seed vigor and field performance of rice (Du and Tuong, 2002; Farooq et al., 2006 a).

In a field trial, seed osmopriming treatments with sulfur $(5 \mathrm{~g} / \mathrm{L})$ and $\mathrm{NaCl}$ resulted in increasing the number of tillers per unit area and consequently 
producing higher number of panicles as evident from the positive correlation found between number of tillers and number of panicles (Table 3). Those treatments have enhanced nutrient supply towards developing panicles, resulting in larger grain size (1000-grains weight, Table 3). Enhanced yield attained from primed seeds might be due to vigorous seedling obtained from those seeds, which produced higher number of panicles bearing tillers and panicle weight (Table 3) comparing to healthy hydroprimed grains (the traditional method; soaking in water for $48 \mathrm{hr}$ ). These results are in accordance in part with those of Hussain et al. (2006) who reported that osmopriming with $\mathrm{NaCl}$ was the most promising priming technique. They stated that $\mathrm{NaCl}$ can enhance the seedling establishment, yield and quality of hybrid sunflower. Singh and Gill (1988) reported that wheat seeds soaked in $1 \%$ sodium bicarbonate solution for 30 min increased the number of ear bearing tillers $/ \mathrm{m}^{2}$ and grain yield. In contrary, soaking of wheat seeds in $5 \%$ $\mathrm{NaHCO}_{3}$ or $\mathrm{NaCl}$ for $30 \mathrm{~min}$ or $24 \mathrm{hr}$ reduced \% emergence, tillers and yield in a field trial.

The number of nematode infected tillers $/ \mathrm{m}^{2}$ increased when nonprimed, nematode-infected grains were used. The white tip nematode negatively affected the shoot/root ratio, plant height, flag leaf area, 1000-grain weight and the crop grain yield. A close relation ship between-white tip nematode mortality in the laboratory and nematode severity percentage in field trials has been found in rice as shown in Tables 2 and 4.

Primed seeds had higher vigor levels (Basra et al., 2002) with earlier start of emergence (Basra et al., 2002 and Farooq et al., 2006 b). Positive correlation between seed vigor and field performance has been found in rice (Yamauchi and Winn, 1996) and sunflower (Hussain et al., 2006).

Osmopriming with all salts in this study and sulfur priming significantly increased the nematode mortality. Osmopriming with Sulfur $(5 \mathrm{~g} / \mathrm{L})$ and $5 \%$ $\mathrm{NaCl}$ showed the most pronounced effect in nematode mortality in the laboratory and enhanced grain yield (Tables 3 and 4). The highest mortality was recorded by soaking seeds in $\mathrm{NaCO}_{3}+\mathrm{NaCl}, 5 \mathrm{~g} / \mathrm{L}$ of Sulfur $80 \% \mathrm{WP}$, $\mathrm{NaCO}_{3}$ and Sulfur+ $\mathrm{MgSO}_{4}$, with no significant differences between these treatments. The high nematode mortality with sodium carbonate in the laboratory may be due to the high alkalinity $(\mathrm{pH}=10.25)$. The Sulfur effect may have another mechanism than the osmopriming. The excessive concentration of hydrogen sulfide in the solution may have a killing effect on the white tip nematode after its release from the infected grain. Young rice plants are particularly susceptible to sulfide toxicity which was observed with the $10 \mathrm{~g} / \mathrm{L}$ of sulfur as recorded on the seedling vigor index and shoot/ratio (Table 2). Physiological disorders attributed to $\mathrm{H}_{2} \mathrm{~S}$ toxicity may affect the seedling growth and white tip nematode.

In conclusion, in the field trial, the effect of priming with sulfur and osmohardining with $\mathrm{NaCl}$ on grain yield and its components is evidenced first in the better seedling establishment, plant height, flag leaf area, number of tillers and panicles $/ \mathrm{m}^{2}$ and the weight of 1000 -grains that allow some protection from other biotic and abiotic stresses. Priming seems to positively affect all yield components. 
These studies can be supportive to smallholder farmers with social and environmental benefits that can help control programs of rice seed-borne diseases such as white tip nematodes and foot rot disease (bakanae disease). Use of seed priming as a preventive treatment to manage foliar nematodes may provide an environmentally safe alternative to excessive use of nematicides. We found that sulfur and $\mathrm{NaCl}$ treatment consistently reduced the numbers of nematode-infected leaves and the size of chlorotic lesions on Rieho cultivar as well as the percentage of bakanae disease.

\section{REFERENCES}

Abdel Hadi, M. A., E. I. El-Shafeey, M. S. Tadros and M. H. Soliman (2005). Occurrence distribution and epidimics of white tip nematode (Aphelenchoides besseyi) on rice plants in Egypt. J. Agric. Sci. Mansoura Univ., 30(2):1169-1182.

Ashraf, M. and , M.R. Foolad (2005). Pre-sowing seed treatment-a shotgun approach to improve germination, plant growth, and crop yield under saline and non-saline conditions. Adv. Agron. 88:223-271.

Ashraf, M., N. Akhtar, F. Tahira and F. Nasim (1999). Effect of $\mathrm{NaCl}$ pretreatment for improving seed quality in cereals. Seed Sci. Technol., 20: $435-440$.

Basra, S.M.A., Zia, M.N., Mehmood, T., Afzal, I. and A. Khaliq (2002). Comparison of different invigoration techniques in wheat (Triticum aestivum L.) seeds. Pak. J. of Arid Agric. 5:11-16.

Basra, S.M.A., Farooq M. and A. Khaliq (2003). Comparative study of presowing seed enhancement treatments in treatments in fine rice (Oryza sativa L.). Pak. J. Life Soc. Sci., 1(1):5-9.

Basra, S.M.A., Farooq, M., Hafeez, and K., N. Ahmad (2004). Osmohardening: a new technique for rice seed invigoration. Inter. Rice Res. Notes 29:80-81

Basra,S.M.A., M. Farooq and R. Tabassum (2005). Physiological and biochemical aspects of seed vigor enhancement treatments in fine rice (Oryza sativa L.). Seed Sci. Technol., 33: 623-628.

Bradford K.J. (1986). Manipulation of seed water relations via osmotic priming to improve germination under stress conditions. Hort. Sci. 21:1105-1112.

Bradford K.J., Steiner, J.J. and S.E. Trawatha (1990). Seed priming influence on emergence and emergence of pepper seed lots. Crop Sci. 30:718721.

Bray, C.M. (1995). Biochemical process during the osmopriming of seeds. In: Seed Development and Germination, (eds.). Kigel, I. and G. Galili. Marcel Dekker, New York. pp: 767-789.

Dell'Aquilla, A. and V. Tritto (1990). Ageing and osmotic priming in wheat seeds: effects upon certain components of seed quality. Ann. Bot. 65:21-26. 
Du, L.V. and T.P. Tuong (2002). Enhancing the performance of dry-seeded rice: effects of seed priming, seedling rate, and time of seedling. In: Direct Seeding: Research Strategies and Opportunities. (eds.), Pandey, S. M. Mortimer, L. Wade, TP. Tuong, K. Lopes and B. Hardy. International Rice Research Institute, Manila, Philippines, pp: 241-256.

Duncan, D.B. (1955). Multiple range and multiple F test. Biometrics, 11:1-42.

EL-Shafey, R.A.S. (2007). Biological and ecological studies on white tip nematode in rice with special reference to its control. Ph.D. Thesis, Fac. Agric., Kafr EL-Sheikh University .

Farooq, M., S.M.A. Basra, Karim and H.A., Afzal (2004). Optimization of seed hardening techniques for rice seed invigoration. Emirates J. Agric. Sci. 16:48-57

Farooq, M., S.M.A. Basra and N. Ahmed (2005a). Rice seed priming. Int. Rice Res. Notes 30:45-48

Farooq, M., S.M.A. Basra and K. Hafeez (2005b). Seed invigoration by osmohardening in indica and japonica rice. Seed Sci. Technol. 33(3):.

Farooq, M., S.M.A. Basra, R.Tabassum, and I. Afzal (2006a). Enhancing the performance of direct seeded fine rice by seed priming. Plant Prod. Sci. 9:446-456

Farooq, M., S.M.A. Basra and K. Hafeez (2006b). Seed invigoration by osmohardening in coarse and fine rice. Seed Sci Technol 34:181-187.

Farooq, M., S.M.A. Basra, and M.A., Afzal (2006c). Integration of pre-sowing soaking, chilling and heating treatments for vigor enhancement in rice (Oryza sativa L.). Seed Sci. Technol. 34:499-506.

Farooq, M., S.M.A. Basra, and A. Wahid (2006d). Priming of field-sown rice seed enhances germination, seedling establishment, allometry and yield. Plant Growth Regul. 49:285-294.

Farooq, M., S.M.A. Basra, N. Ahmad, and G. Murtaza (2009). Enhancing the performance of transplanted coarse rice by seed priming. Paddy Water Environ. 7:55-63.

Giudici, M.L., B.Villa, A. M. Collegarin and L. Tamborini (2003). white tip disease in Italian rice. Proceeding of the 3rd International Rice Conference, 10-13 March, Punta de L"Este, Uruguay.

Gomez, K.A. and A.A. Gomez (1984). Statistical procedures for Agricultural Research. Second Edition. John Wiley \& Sons, New York.

Harris, D., R.S. Tripathi and A. Joshi (2002). On-farm seed priming to improve crop establishment and yield in dry direct-seeded rice In: Direct Seeding: Research Strategies and Opportunities. (eds.). Pandey, S., M. Mortimer, L. Wade, T.P. Tuong, K. Lopes and B. Hardy. International Research Institute, Manila, Philippines, pp: 231-240.

Hussain, M., Farooq M., Shahzad M.A. Basra and N. Ahmad (2006). Influence of seed priming techniques on the seedling establishment, yield and quality of hybrid sunflower. International Journal of Agriculture \& Biology (8) 1.

Huynh, V. N., Pham V. D., S.B., Mathur (2001). Effect of cleaning on seed health and seed germination of rice. OMonRice 9: 138-139 
Lee, S.S., J.H. Kim, 1999. Morphological change, sugar content, and amylase activity of rice seeds under various priming conditions. Korean J. Crop. Sci. 44:138-142

Lee, S.S. and J.H. Kim (2000). Total sugars, a-amylase activity, and emergence after priming of normal and aged rice seeds. Korean $\mathrm{J}$. Crop. Sci. 45:108-111.

Musa. A. M., D. Harris, C. Johansen and J. Kumar (2001). Short duration chickpea to replace fallow after aman rice: the role of on-farm seed Priming in the high barind tract of bangladesh. Expl. Agric. 37, pp. 509521

Ou, S.H. (1985). Rice Diseases. 2nd ed., Kew, England, Commonwealth Mycological Institute. 247-256.

Rajan, L. A. and A. Lal (2006). Extraction of Aphelenchoides besseyi from rice. Nematropica, 36(1): 1-6.

Rood, M.A. (2004). Bakanae in-field yield loss. Rice J. 15: 8-10.

Tang, Q.Y. and Feng, M.G. (1997). Practical Statistics and DPS Data Processing System. China Agricultural Press, Beijing. 1- 407.

RRTC, (2008). Rice Research and Training Center. Annual Rice National Campaign Report of Rice Program, Agric. Res. Center, Ministry of Agriculture and Land Reclamation, Egypt.

RRTC, (2009). Rice Research and Training Center. Annual Rice National Campaign Report of Rice Program, Agric. Res. Center, Ministry of Agriculture and Land Reclamation, Egypt.

Ruan, S., Q. Xue and K. Tylkowska (2002). The influence of priming on germination of rice (Oryza saliva L.) seeds and seedling emergence and performance in flooded soils. Seed Sci. Technol., 30: 61-67.

Singh, H. and H.S. Gill (1988). Effect of seed treatment with salts on germination and yield of wheat. Agric. Sci. Digest, 8: 173-5

Yamauchi, M. and T. Winn (1996). Rice seed vigor and seedling establishment in anaerobic soil. Crop Sci., 36: 680-686

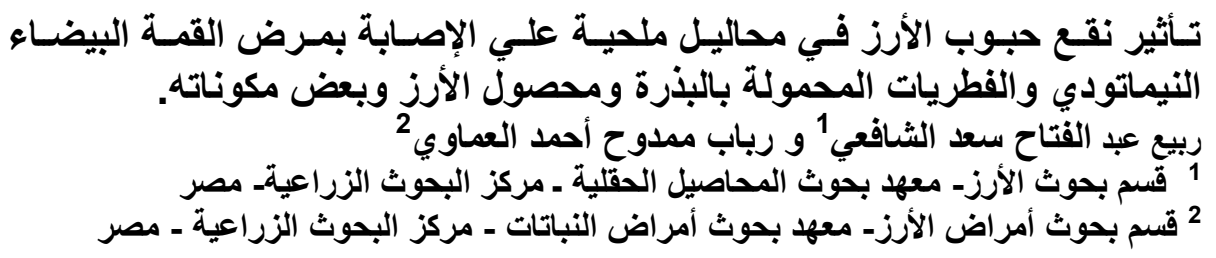

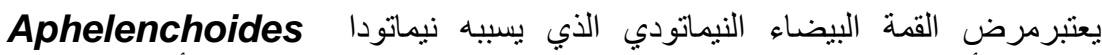

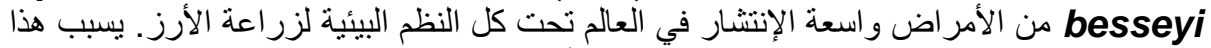

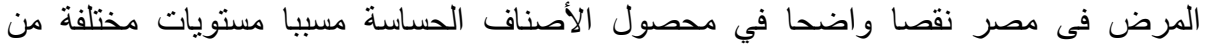

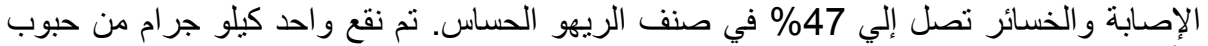

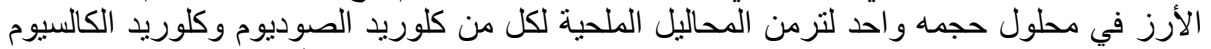

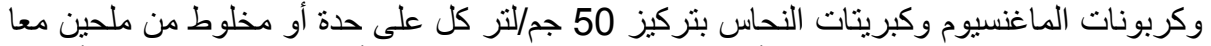

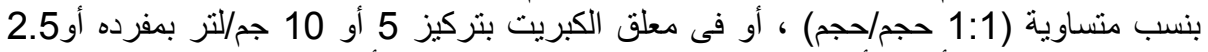
جم/لتز مخلوطا مع أحد الأملاح. وكبريتات النحاس 1.5 جم/لتز أو2.5 جم/لتر وذللك لمدة 48 


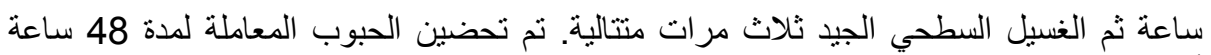

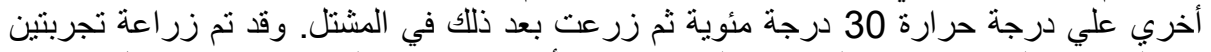

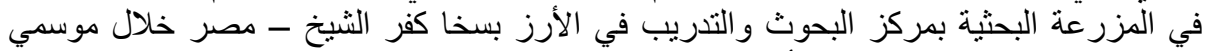

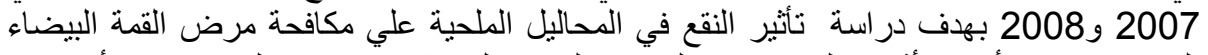

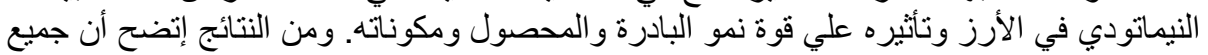

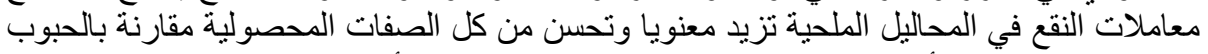

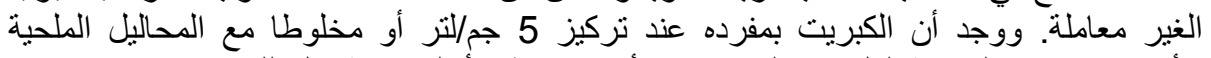

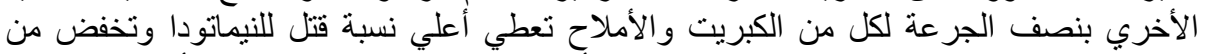

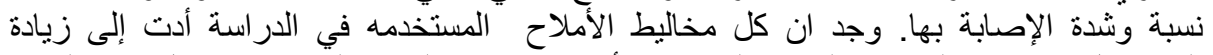

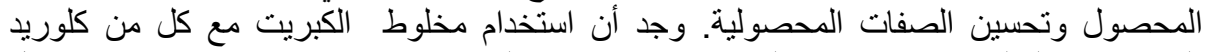

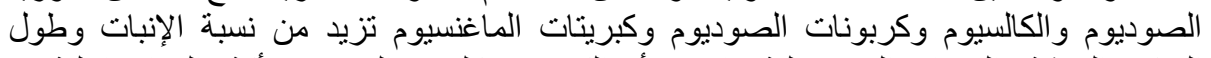

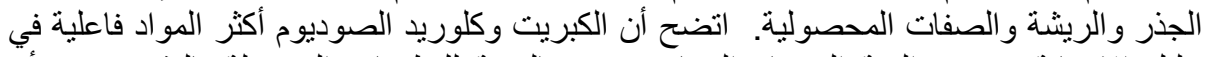

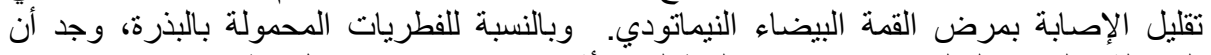
Fusarium المعاملة بكلوريد الكالسيوم بمفرده ومخلوط لديه تأثير إيجابي في إستئصال فطريات Moniliforme, Bipolaris oryzae, and Alternaria padwickii

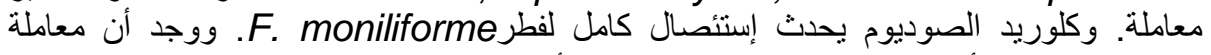

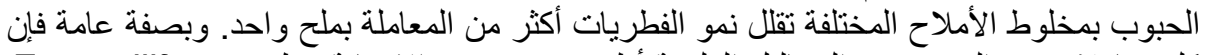

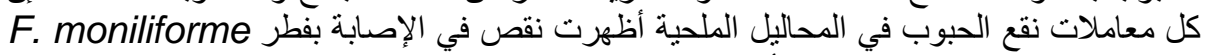

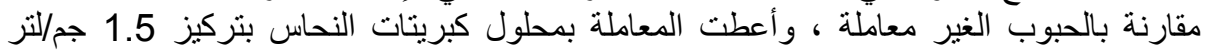
حماية كاملة من الفطريات المحمولة بالبذرة.

$$
\text { كلية الزراعة البحوث جامعة الزئصورة }
$$

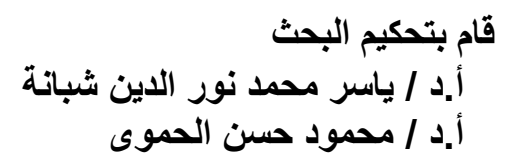

